\title{
An Asynchronous Two-Way Relay System with Full Delay Diversity in Time-Varying Multipath Environments
}

\author{
Ahmad Salim $^{\dagger}$ and Tolga M. Duman ${ }^{\dagger \ddagger}$, Fellow, IEEE \\ †School of ECEE, Arizona State University, Tempe, AZ, USA, E-mails: \{assalim,duman\}@asu.edu \\ Department of EEE, Bilkent University, Bilkent, 06800, Ankara, Turkey, E-mail: duman@ee.bilkent.edu.tr
}

\begin{abstract}
We consider design of asynchronous OFDMbased diamond two-way-relay (DTWR) systems in time-varying frequency-selective (doubly-selective) fading channels such as underwater acoustic (UWA) channels. In a DTWR channel, two users exchange their messages with the help of two relays. Most of the existing work on asynchronous DTWR systems assume only small relative propagation delays between the received signals at each node. However, in practical systems, significant delays may take place. Our proposed system is able to tolerate the delay even if it exceeds the length of the OFDM block which is almost inevitable in UWA channels. We provide analytical and numerical results to verify the advantages of the proposed scheme in mitigating large delays in different fading conditions.
\end{abstract}

\section{INTRODUCTION}

An increasing number of UWA research problems have been emerging due to the need for efficient designs of UWA sensor networks and reliable communication between submarines and autonomous underwater vehicles. On the other hand, excessively large delays, time and frequency selectivity makes the UWA channel one of the harshest communication media making it very difficult to satisfy the requirements of these emerging applications [1].

TWR channels [2] have also received increased attention recently due to the bi-directional nature of communication in many existing systems such as cellular networks, satellite communications, indoor wireless communications among others.

Bringing the TWR idea into UWA communications can increase the system's spectral efficiency and overcome coverage problems. However, employing TWR for UWA communications faces many challenges, one of which is considered in this work, that is, with the low speed of the sound signal, large delays among the received signals at a specific node may be experienced. Therefore, having an accurately synchronized DTWR system in a UWA environment is very difficult. Hence, novel schemes are required to face the resulting asynchrony and to ensure reliable communications in such conditions.

To account for the asynchrony caused by having the simultaneously received signals experience different delay spreads, Lu et al. proposed an OFDM-based scheme in [3]. If the maximum of the delay spreads of the simultaneously received signals is within the cyclic prefix (CP), then the effect will disappear in the frequency domain. In [4], a scheme based on sphere decoding is proposed to mitigate the effects of time

Tolga M. Duman is with the Department of Electrical and Electronics Engineering (EEE), Bilkent University, Bilkent, Ankara, 06800, Turkey, and is on leave from the School of Electrical, Computer and Energy Engineering (ECEE) of Arizona State University.

This work was supported in part by the National Science Foundation under the grants NSF-CCF 1117174 and NSF-ECCS 1102357. and phase asynchrony. Two precoding-based schemes were proposed in [5] based on the channel inversion technique. In [6], the authors propose a scheme that jointly mitigates synchronization errors and provides full spatial diversity. The scheme is based on having an appropriate $\mathrm{CP}$ and performing simple operations at the relay which leads to overcoming time asynchrony and obtaining an orthogonal space time block code (OSTBC) structure. In [7], the authors derive a sliding window estimator to find the optimal timing for the discrete Fourier transform (DFT) window. For a UWA-TWR channel, Wang et al. study in [8] three schemes for obtaining network-coded channel-uncoded packets at the relay.

None of the solutions presented above have tackled the problem of large delays among the different signals transmitted. Therefore, motivated by the work in [9] for singleway relay channels, this paper proposes a scheme that can be used in a UWA channel or other channels in which large delays may be experienced. The objective is to design an efficient scheme that does not require an excessively long cyclic prefix and at the same time tolerates any delay with a small increase in complexity. We aim to avoid the "delay within CP" requirement that is generally assumed in the literature of OFDM-based TWR systems, e.g., $[3,4,6]$. Up to the authors' knowledge, the best reported result on this issue is due to [6] which does not provide a general solution to the delay problem as it is limited to delays within the CP of an OFDM block. Our approach to address the large delay issue, similar to [9] for single-way relay channels, is to have the received signal on each subcarrier in a delaydiversity structure. We will show that with proper signaling, using Viterbi algorithm on each subcarrier over the received blocks, delay diversity can be obtained.

The remainder of the paper is organized as follows. Section II gives a description of the channel model. Section III presents the proposed scheme for harnessing delay diversity. Section IV deals with a pair-wise error probability (PEP) analysis for the proposed scheme. Section V provides the simulation results conducted to evaluate benefits of the proposed scheme. Finally, conclusions are drawn in Section VI.

Notation: Bold-capital letters refer to frequency-domain vectors, bold-lower case letters refer to time-domain vectors, capital letters refer to matrices or elements of frequencydomain vectors (according to context), and lower case letters refer to scalars or elements of time-domain vectors. $F$ is the normalized DFT matrix of size- $N$. The subscript $i r$ refers to the channel from node $i$ to node $r, i, r \in\{1,2, A, B\}$ and $A r B$ refers to the $U_{A}-R_{r}-U_{B}$ link. 


\section{Channel Model}

Fig. 1 shows the DTWR system model. The time-varying multipath fading channels are modeled by the discrete channel impulse responses (CIRs) from users to relays $h_{i r, l}^{n}$ and from relays to users $h_{r i, l}^{n}, i \in\{A, B\}, r \in\{1,2\}, n \in \mathbb{Z}^{+}$, $l \in\left\{1,2, \ldots, L_{h_{i r}}\right\}$, where $L_{h_{i r}}$ represents the length of the specified channel. The overall channel response affecting the $n^{\text {th }}$ input sample over the $L_{h_{i r}}$ lags can be expressed as:

$$
\boldsymbol{h}_{i r}(n, \tau)=\sum_{l=1}^{L_{h_{i r}}} h_{i r, l}^{n} \boldsymbol{\delta}\left(\tau-\tau_{i r, l}\right),
$$

where $\tau$ is the lag index and $\tau_{i r, l}$ is the delay of the $l^{\text {th }}$ path normalized by the sampling period $T_{S}$.

We assume $\left\{h_{i r, l}^{n}\right\}_{l \in\left\{1,2, \ldots, L_{h_{i r}}\right\}}$ are complex Gaussian wide sense stationary processes with zero mean and total envelope power of $\sigma_{h_{i r}, l}^{2}$, where they are correlated over the time $(n)$ and independent over the lag $l$. In other words, the channel taps follow independent Rayleigh fading and all taps at the same lag are time-varying and correlated over time. Also, all the channels shown in Fig. 1 are independent from each other.

\section{A DTWR System With Full Delay Diversity BASED ON FULL-DUPLEX RELAYS}

At each user, a standard OFDM modulator with $N$ subcarriers is used. The resulting sequence is appended with a $\mathrm{CP}$ of length $N_{C P}$. Each user sequentially transmits $M$ blocks each of length $N+N_{C P}$ where the $M$ consecutive blocks comprise one frame and the consecutive frames are separated by a sufficient guard time that ensures no frame affects another.

We assume system-wide simultaneous transmission and reception due to full-duplex operation at all nodes. However, we model their operation in two phases: the multiple access (MAC) phase and the broadcast (BC) phase. During the MAC phase, users simultaneously transmit their messages to the relay. In the BC phase, analog network coding (ANC) is performed. In ANC, the relay broadcasts an amplified version of its received signal [10]. Since we use ANC in conjunction with full-duplex nodes, we refer to this scheme as ANC-FD.

In an asynchronous DTWR system operating over a multipath channel, two types of timing errors may exist [6]. The first type is due to the misalignment of the users' signals at one relay in the MAC phase as shown in Fig. 2 where $\mathbf{y}_{i r}$, $i \in\{A, B\}, r \in\{1,2\}$, is the portion of the signal received at the $r^{\text {th }}$ relay that corresponds to the message of user $i$ after passing through the channel in the MAC phase. The second type takes place when the signals sent by the two relays in the $\mathrm{BC}$ phase arrive at different times at one user.

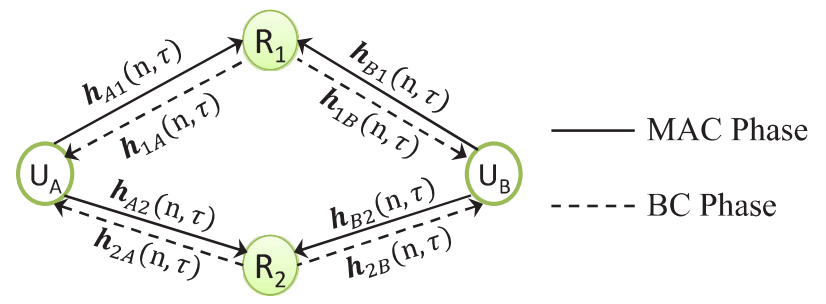

Fig. 1: The DTWR system model.

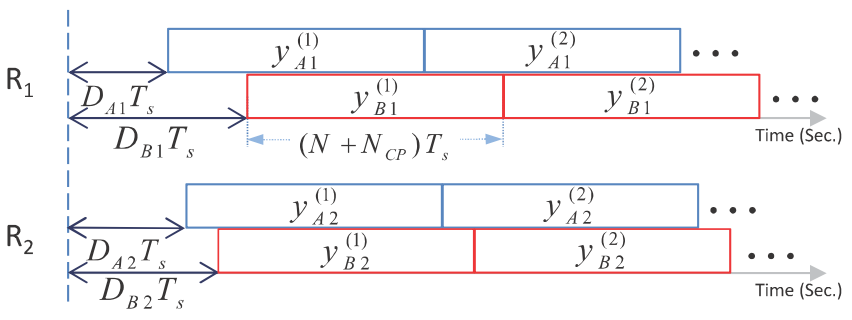

Fig. 2: Signal structure at the two relays.

In a conventional TWR system with a single relay, upon receiving the relay's signal each user will subtract its contribution and detect its partner's message. However, in our case where we have two relays, each user removes its selfinterference which consists of two faded copies of its own signal. After that, each user employs the Viterbi algorithm to extract delay diversity out of the two copies of its partner's signal.

Let $D_{i j}, i, j \in\{A, B, 1,2\}$ where $i \neq j$, denote the propagation delay experienced from node $i$ to node $j$. We assume that the users have full knowledge of the channels and the delays they require, for instance, user $B$ requires all the channels except $\boldsymbol{h}_{1 A}(n, \tau)$ and $\boldsymbol{h}_{2 A}(n, \tau)$ and all the delays except $D_{1 A}$ and $D_{2 A}$. We also assume, without loss of generality, that $D_{B r}>D_{A r}, r \in\{1,2\}$ and $D_{i 2}>D_{i 1}, i \in\{A, B\}$.

\section{A. Minimum CP Length}

Let $L_{i}=\max _{r \in\{1,2\}}\left(L_{h_{i r}}+L_{h_{r i^{\prime}}}\right), i, i^{\prime} \in\{A, B\}, i \neq i^{\prime}$, denote the maximum delay spread over all links from user $i$ to user $i^{\prime}$, $i, i^{\prime} \in\{A, B\}$. The minimum cyclic prefix applied at user $i$ that is required to jointly mitigate the effect of the timing errors and multipath channel can be extended from [9, Sec. III-A] as $N_{C P, i} \geq N+2 L_{i}$. We choose $N_{C P, i}=N+2 L_{i}$ in the remainder of the paper. We assume symmetry between the two users and hence, we can drop the user index and write $L$ and $N_{C P}$ instead of $L_{i}$ and $N_{C P, i}$, respectively. Note that $N_{C P}$ does not depend on the delay; it only depends on the number of subcarriers and length of the channels. This actually explains why our ANC-FD scheme surpasses the systems in [6] and [3] as we will study in detail in Section V.

\section{B. Relay Processing}

The data vector representing the frequency-domain message of the $i^{t h}$ user, $i \in\{A, B\}$, during the $m^{\text {th }}$ block is denoted by $\mathbf{X}_{i}^{(m)}$ where $\mathbf{X}_{i}^{(m)}=\left[X_{i, 1}^{(m)}, X_{i, 2}^{(m)}, \ldots, X_{i, N}^{(m)}\right]^{T}$. Taking the IDFT, we obtain $\mathbf{x}_{i}^{(m)}=\operatorname{IDFT}\left(\mathbf{X}_{i}^{(m)}\right)$ where $\mathbf{x}_{i}^{(m)}=$ $\left[x_{i, 1}^{(m)}, x_{i, 2}^{(m)}, \ldots, x_{i, N}^{(m)}\right]^{T}$. The transmitted signal from the $i^{t h}$ user during the $m^{\text {th }}$ block, $i \in\{A, B\}$, is given by $\mathbf{x}_{T, i}^{(m)}=$ $\sqrt{P_{i}} \zeta\left(\mathbf{x}_{i}^{(m)}\right)$ where $\mathbf{x}_{T, i}^{(m)}=\left[x_{T, i, 1}^{(m)}, x_{T, i, 2}^{(m)}, \ldots, x_{T, i, N+N_{C P}}^{(m)}\right]^{T}, P_{i}$, $i \in\{A, B\}$, is the transmission power at the $i^{\text {th }}$ user and $\zeta(\cdot)$ is an operator that appends a length- $N_{C P}$ cyclic prefix. Upon reception, the $r^{\text {th }}$ relay uses ANC by simply amplifying its received superimposed signal denoted by $\mathbf{y}_{r}^{(m)}$ for the $m^{\text {th }}$ block. The transmitted signal from the $r^{t h}$ relay is given by $\mathbf{x}_{r}^{(m)}=\sqrt{P_{r}} \mathbf{y}_{r}^{(m)}, r \in\{1,2\}$, where $P_{r}, r \in\{1,2\}$, is the transmit power at the $r^{\text {th }}$ relay. 


\section{End-User Processing}

We now discuss the processing at user B. Similar arguments can be stated for user A due to symmetry. Fig. 3 shows the structure of the received signal at user B after the selfinterference is removed, that is $\mathbf{y}_{A B, e}$. Since the frame relayed by $R_{2}$ is received $D_{A B}$ samples after that relayed by $R_{1}$ where $D_{A B}=\left(D_{A 2}+D_{2 B}\right)-\left(D_{A 1}+D_{1 B}\right), \mathbf{y}_{A B, e}$ can be written as

$$
\mathbf{y}_{A B, e}=\left[\mathbf{y}_{A 1 B, e}^{T}, \mathbf{0}_{D_{A B}}^{T}\right]^{T}+\left[\mathbf{0}_{D_{A B}}^{T}, \mathbf{y}_{A 2 B, e}^{T}\right]^{T}+\mathbf{w}_{B},
$$

where $\mathbf{y}_{A r B, e}, r \in\{1,2\}$, is the portion of $\mathbf{y}_{A B}$ relayed by the $r^{t h}$ relay and $\mathbf{w}_{B}$ is the noise vector at the $r^{\text {th }}$ relay whose entries are assumed to be independent and identically distributed (iid) circularly-symmetric complex Gaussian random variables with zero mean and variance of $\sigma_{B}^{2}$. The residual delay between the overlapping blocks of $\mathbf{y}_{A 1 B, e}$ and $\mathbf{y}_{A 2 B, e}$ in samples is denoted by $d_{A B}$ where $d_{A B}=\bmod \left(D_{A B}, 2 N+2 L\right)$. Define $B D_{A B}$ as the effective OFDM block delay observed at user B between the blocks from user A, for instance, $B D_{A B}=2$ in Fig. 3. More generally, $B D_{A B}=\left\lfloor\frac{D_{A B}}{2 N+2 L}\right\rfloor+\left\lfloor\frac{d_{A B}}{N+L}\right\rfloor$.

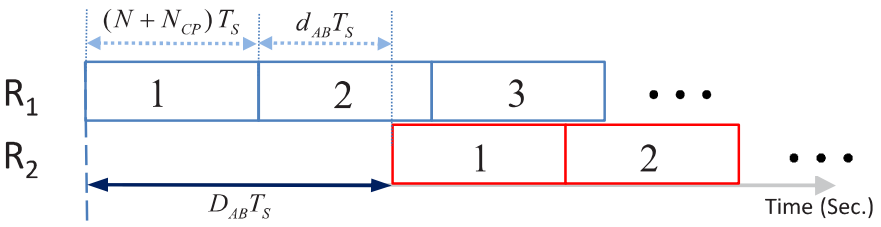

Fig. 3: The structure of $\mathbf{y}_{A B, e}$ with one block delay.

Let $H_{t l, i r}^{\left(m_{r}\right)}, i \in\{A, B\}, r \in\{1,2\}$ and $m_{r} \in\{1,2, \ldots, M+$ $\left.B D_{r}\right\}$ denote the time-lag channel matrix that represents the time-domain effect of circular convolution of $\mathbf{x}_{i}^{\left(m_{r}\right)}$ with $\boldsymbol{h}_{i r}(n, \tau)$ for all the $N$ samples in the selected window during the $m_{r}{ }^{\text {th }}$ block [11]. Let $P_{A r}=\sqrt{P_{A} P_{r}}, r \in\{1,2\}$. Without loss of generality, we assume $d_{A B}<N+L$. The other case is only different in terms of the resulting phase shift in frequency domain. After selecting the window of length $N+L$ as described in [9] and discarding the first $L$ samples, the resulting $N$-sample OFDM blocks, $\left\{\mathbf{y}_{A B}^{(m)}\right\}_{m=1}^{M+B D_{A B}}$, can be written as:

$$
\mathbf{y}_{A B}^{(m)}=P_{A 1} H_{t l, A 1 B}^{(m)} \mathbf{x}_{A}^{(m)}+P_{A 2} \Psi_{d_{A B}} H_{t l, A 2 B}^{\left(m-B D_{A B}\right)} \mathbf{x}_{A}^{\left(m-B D_{A B}\right)}+\mathbf{v}_{B}^{(m)},
$$

where $\mathbf{x}_{A}^{(m)}=\mathbf{0}_{N}$ for $m<1$ and $m>M, H_{t l, A r B}^{(m)}=H_{t l, r B}^{(m)} H_{t l, A r}^{(m)}$, $r \in\{1,2\}$ is the equivalent time-lag channel matrix corresponding to the link from user A through the $r^{\text {th }}$ relay to user B. The vector $\mathbf{v}_{B}^{(m)}$ is simply the part of $\mathbf{w}_{B}$ in the considered window. $\Psi_{d_{A B}}$ is a circulant matrix of size $N \times N$ whose first column is given by $\psi_{d_{A B}}=\left[\mathbf{0}_{d_{A B}}^{T}, 1, \mathbf{0}_{N-d_{A B}-1}^{T}\right]^{T}$.

After performing DFT on $\mathbf{y}_{A B}^{(m)}$ by an $N$-point DFT module, the frequency-domain block can be written as

$$
\begin{aligned}
\mathbf{Y}_{A B}^{(m)} & =D F T\left(P_{A 1} H_{t l, A 1 B}^{(m)} \mathbf{x}_{A}^{(m)}+P_{A 2} \Psi_{d_{A B}} H_{t l, A 2 B}^{\left(m-B D_{A B}\right)} \mathbf{x}_{A}^{\left(m-B D_{A B}\right)}+\mathbf{v}_{B}^{(m)}\right) \\
& =P_{A 1} H_{d f, A 1 B}^{(m)} \mathbf{X}_{A}^{(m)}+P_{A 2}\left(H_{d f, A 2 B}^{\left(m-B D_{A B}\right)} \mathbf{X}_{A}^{\left(m-B D_{A B}\right)}\right) \circ \mathbf{g}_{d_{A B}}+\mathbf{V}_{B}^{(m)},
\end{aligned}
$$

where $\circ$ denotes the Hadamard product, $\mathbf{V}_{B}^{(m)}=F \mathbf{v}_{B}^{(m)}, \mathbf{g}_{d_{A B}}=$ $\left[1, e^{-j \frac{2 \pi d_{A B}}{N}}, \ldots, e^{-j \frac{2 \pi d_{A B}(N-1)}{N}}\right]^{T}$ and $H_{d f, A r B}^{(m)}=F H_{t l, A r B}^{(m)} F^{H}$ is the effective Doppler-frequency channel matrix for the $m^{\text {th }}$ block over the $U_{A}-R_{r}-U_{B}$ link [11]. For block fading channels that are constant within each block but vary from one block to another, $H_{t l, A r B}, r \in\{1,2\}$, have a circulant structure making $H_{d f, A r B}, r \in\{1,2\}$ diagonal which means that there is no intercarrier interference (ICI). When the channel is time-varying within the same OFDM block, neither $H_{t l, A r B}$ will be circulant nor will $H_{d f, A r B}$ be diagonal, which means that the subcarrier orthogonality is lost, giving rise to ICI. Here, we do not investigate ICI mitigation, instead we ignore the effects of the off-diagonal elements of $H_{d f, A r B}^{(m)}$ and concentrate on solving the issue of long delays between the blocks received from the two relays in DTWR systems.

Let $V_{B, k}^{(m)}$ denote the $k^{t h}$ element of $\mathbf{V}_{B}^{(m)}$ and $q_{r, k}^{(m)}=$ $\sqrt{P_{A} P_{r}}\left[H_{d f, A r B}^{(m)}\right]_{k, k}$. By discarding the off-diagonal elements of $H_{d f . A r B}^{(m)}$, the received signal on the $k^{t h}$ subcarrier of the $m^{t h}$ block can be written as

$$
Y_{A B, k}^{(m)}=q_{1, k}^{(m)} X_{A, k}^{(m)}+e^{-j \frac{2 \pi(k-1) d_{A B}}{N}} q_{2, k}^{\left(m-B D_{A B}\right)} X_{A, k}^{\left(m-B D_{A B}\right)}+V_{B, k}^{(m)} .
$$

D. Subcarrier Diversity for Small Delays

The proposed system provides a diversity gain up to $N_{R}$, where $N_{R}$ is the number of relays used. However, this gain is dependent upon having at least one block delay. To provide a diversity gain for smaller delays, where $B D_{A B}=0$, we propose a modification to the original system by using the modulation property of DFT which enables a diversity order of up to $N_{R}$ as long as $N_{R} \leq N$. In this case, the $r^{\text {th }}$ relay does not only amplify its received signal but it also multiplies the $n^{\text {th }}$ sample, $n \in$ $\left\{1, \ldots, N+N_{C P}\right\}$, by $e^{j \frac{2 \pi(n-1)(r-1)}{N}}$, which will have the effect of having a circular shift of $r-1$ samples in the frequency domain. We only consider small delays in which $D_{A B} \leq N+L$. Now, discarding the off-diagonal elements of $H_{d f, A r B}^{(m)}$ as in (5) and assuming that $N_{R} \leq N, Y_{A B, k}^{(m)}$ can be written as

$$
Y_{A B, k}^{(m)}=q_{1, k}^{(m)} X_{A, k}^{(m)}+\sum_{r=2}^{N_{R}} e^{-j \frac{2 \pi(k-1) D_{A B}}{N}} q_{r, k}^{\left(m-B D_{A B}\right)} X_{A,<k-r+1>_{N}}^{\left(m-B D_{A B}\right)}+V_{B, k}^{(m)},
$$

where $<\cdot>_{N}$ is the cyclic shift operator defined as

$$
<l>_{N}= \begin{cases}N+l, & l \leq 0 \\ l, & l>0 .\end{cases}
$$

and $q_{r, k}^{(m)}=\left[\theta_{r}\left(H_{d f, r B}^{(m)}\right) H_{d f, A r}^{(m)}\right]_{k, k}$ where $\theta_{r}(\cdot)$ circularly shifts the rows of its argument by $(r-1)$ rows downward.

\section{E. Detection of Partner's Message}

The structure of the received signal in (5) on the $k^{\text {th }}$ subcarrier from all blocks is similar to a single-carrier (SC) intersymbol interference (ISI) channel or equivalently to a multiinput single-output (MISO) system applying delay diversity. As noted in [9], (5) has a delay diversity structure which can be used to improve the system performance. For our system, after the reception of $M+B D_{A B}$ blocks, each user implements $N$ parallel Viterbi decoders of $M_{c}^{B D_{A B}}$ states where $M_{c}$ is the constellation size. Each Viterbi decoder takes the collected received samples for one subcarrier over the $M+B D_{A B}$ blocks 
and detects the signals transmitted for this subcarrier over the $M$ blocks. For $B D_{A B}=0$, unlike the large delay case, we observe a delay structure among the symbols on different subcarriers of the same block as in (6). Hence, the detection is performed on a block-by-block basis, and for each of the $M$ blocks, the receiver drops the first $\left(N_{R}-1\right)$ symbols and uses a Viterbi detector with $M_{c}^{N_{R}-1}$ states. The spectral efficiency loss due to the partial symbol drop would be negligible if $N_{R} \ll N$ which is the case in practice.

\section{F. Maximum Achievable Data Rate}

Let $\eta$ denote the maximum achievable data rate when BPSK modulation is used. For the transmission of one OFDM block in the ANC-FD system, we require $N+N_{c p}$ samples. Hence, the rate of ANC-FD can be written as

$$
\eta_{A N C-F D}=\frac{M}{M+B D_{A B}} \cdot \frac{N}{N+N_{C P}} .
$$

From (8), we can see that the only effect of the delay on $\eta_{A N C-F D}$ is through the number of block delays $B D_{A B}$ rather than the actual delay $D_{A B}$; an effect that is negligible if $M$ is sufficiently large. To see the advantage of the proposed scheme, we compare it to other schemes that solve the asynchrony issue. We consider the ANC-STBC scheme in [6] and the ANC-OFDM scheme which is an extension of the system in [3] to the dual-relay case. Noting that the rate for both ANCSTBC and ANC-OFDM is given by $\frac{N}{2 N+N_{C P, M A C}+N_{C P B C}}$, where $N_{C P, M A C}$ and $N_{C P, B C}$ are the minimum CP lengths required for the $\mathrm{MAC}$ and $\mathrm{BC}$ phases, respectively, we can see that the rate is affected significantly with the increase of the propagation delays due to the increase of either $N_{C P, M A C}, N_{C P, B C}$ or both.

\section{PAIR-Wise ERror Probability Analysis}

Study of the proposed system's PEP can give an insight to the diversity order and provide a basis for code design. With this motivation, here we consider binary phase-shift keying (BPSK) and assume that the MAC phase links experience much higher signal-to-noise ratios (SNRs) than those during the $\mathrm{BC}$ phase which allows us to discard the effect of the relays' noise. We assume an independent block fading scenario in which the multipath channel gains are fixed within each block and change independently from one block to the next. Further, we assume $P_{A}=P_{1}=P_{2}=1$. To analyze the PEP, we follow a similar approach to the one in $[9,12]$, however, our channel model is different.

Define $\boldsymbol{w}_{f}(k)=\left[1, e^{-j \frac{2 \pi(k-1)}{N}}, \ldots, e^{-j \frac{2 \pi(k-1)(L-1)}{N}}\right]^{T}$ and $d_{k}^{m}=$ $X_{A, k}^{(m)}-X_{A, k}^{\prime(m)}$. Let $\boldsymbol{d}_{k}(m)=\left[d_{k}^{m}, d_{k}^{m-B D_{A B}}\right]^{T}$ and $\boldsymbol{W}_{f}(k)=$ $\operatorname{Bdiag}\left\{\boldsymbol{w}_{f}(k), \boldsymbol{w}_{f}(k)\right\}$ where Bdiag $\{$.$\} returns the block di-$ agonal matrix formed by the matrices in its argument. Let $D_{A}^{(m)}\left(\boldsymbol{X}_{A,(k)}, \boldsymbol{X}_{A,(k)}^{\prime}\right)=\boldsymbol{W}_{f}(k) \boldsymbol{d}_{k}(m) \boldsymbol{d}_{k}^{H}(m) \boldsymbol{W}_{f}^{H}(k)$, we write

$$
D_{A}^{(m)}\left(\boldsymbol{X}_{A,(k)}, \boldsymbol{X}_{A,(k)}^{\prime}\right)=\boldsymbol{U}_{k, m} \boldsymbol{\Lambda}_{k, m} \boldsymbol{U}_{k, m}^{H},
$$

where $\boldsymbol{U}_{k, m}$ is a unitary matrix and $\boldsymbol{\Lambda}_{k, m}=$ $\operatorname{diag}\left\{\lambda_{k, m, 1}, \ldots, \lambda_{k, m, r_{m}}, 0, \ldots, 0\right\} \quad$ where $\quad\left\{\lambda_{k, m, i}\right\}_{i=1}^{r_{m}}$ are the positive eigenvalues of $D_{A}^{(m)}\left(X_{A,(k)}, X_{A,(k)}^{\prime}\right)$. The PEP of the ANC-FD scheme is upper bounded as ${ }^{1}$

$$
\begin{array}{r}
P\left(\mathbf{X}_{A,(k)} \rightarrow \mathbf{X}_{A,(k)}^{\prime}\right) \leq \frac{1}{2} \prod_{m=1}^{M+B D_{A B}} \prod_{c=1}^{r_{m}}\left[\frac { 4 \sigma _ { B } ^ { 2 } } { \lambda _ { k , m , c } \theta _ { G } ^ { k _ { G } } } \left(\theta_{G} \boldsymbol{\delta}\left(k_{G}-2\right)\right.\right. \\
\left.\left.+(-1)^{k_{G}-2}\left(\frac{4 \sigma_{B}^{2}}{\lambda_{k, m, c}}\right)^{k_{G}-1} \frac{4 \sigma_{B}^{2}}{e^{\lambda_{k, m, c} \theta_{G}^{k_{G}}}} E i\left(-\frac{4 \sigma_{B}^{2}}{\lambda_{k, m, c} \theta_{G}^{k}}\right)\right)\right]
\end{array}
$$

where

$k_{G}= \begin{cases}2, & \sigma_{\kappa, k, m, c, 1}^{2} \sigma_{\mu, k, m, 1}^{2}=\sigma_{\kappa, k, m, c, 2}^{2} \sigma_{\mu, k, m, 2}^{2}, \\ 1, & \text { else , }\end{cases}$

$\theta_{G}= \begin{cases}\sigma_{\kappa, k, m, c, 1}^{2} \sigma_{\mu, k, m, 1}^{2}, & \sigma_{\kappa, k, m, c, 1}^{2} \sigma_{\mu, k, m, 1}^{2}=\sigma_{\kappa, k, m, c, 2}^{2} \sigma_{\mu, k, m, 2}^{2}, \\ \sigma_{\kappa, k, m, c, r_{N Z}}^{2} \sigma_{\mu, k, m, r_{N Z}}^{2}, & \text { else, }\end{cases}$

$E i($.$) is the exponential integral function defined as E i(x)=$ $-\int_{-x}^{\infty} \frac{e^{-t}}{t} d t, \delta($.$) is the Dirac delta function, \sigma_{\mu, k, m, r}^{2}=$ $\sum_{l=1}^{L} \sigma_{h_{A r}, l}^{2}, \sigma_{\kappa, k, m, c, r}^{2}=\sum_{l=1}^{L}\left|U_{k, m, c,(r-1) L+l}\right|^{2} \sigma_{h_{r B}, l}^{2}, U_{k, m, c, l}$ is the $l^{\text {th }}$ element of the $c^{\text {th }}$ column of $\boldsymbol{U}_{k, m}$, and $r_{N Z}$ is the index, $r$, of the nonzero $\sigma_{\kappa, k, m, c, r}^{2}$.

\section{Performance eValuation}

In this section, we evaluate the performance of the proposed scheme at user B in terms of the average bit error rate (BER). Define the SNR at user $i$ while aiming to detect the signal of user $i^{\prime}$ as $S N R_{i}=\frac{P_{i^{\prime}}\left(P_{1}+P_{2}\right)}{\sigma_{i, e f f}^{2}}, i, i^{\prime} \in\{A, B\}, i^{\prime} \neq i$ where $\sigma_{i, e f f}^{2}=P_{1} \sigma_{1}^{2}+P_{2} \sigma_{2}^{2}+\sigma_{i}^{2}$ is the effective noise variance at user $i$. We consider UWA-like sparse Rayleigh multipath fading channels ${ }^{2}$ under two cases: quasi-static or time-varying with the coefficients generated using Jakes' model. We assume $P_{A}=P_{1}=P_{2}=1$ and $\sigma_{B}^{2}=\sigma_{1}^{2}=\sigma_{2}^{2}$, therefore $S N R_{B}=\frac{2}{3 \sigma_{B}^{2}}$. We also assume $M=10, N=64, D_{A 1}=D_{A 2}=D_{1 B}=0, D_{B 1}=48$, $D_{B 2}=56$ and the total occupied bandwidth is $3 \mathrm{kHz}$.

In Fig. 4, we compare our novel ANC-FD scheme with the ANC-STBC scheme of Li et al. [6] and the ANC-OFDM scheme based on [3]. Note that we use two relays for all the three schemes. We also impose equal power and rate for a fair comparison in terms of power, temporal and spectral resources. The assumed modulation for the ANC-FD scheme is Quadrature PSK (QPSK). For the other two schemes, longer $\mathrm{CP}$ is required while experiencing larger delays and hence we increase the size of their constellations in order to maintain the same rate as with our ANC-FD scheme (refer to Section III-F for the data rate expressions). For the duplexing method, the schemes in $[3,6]$ use half-duplex nodes while our proposed scheme uses full-duplex nodes and hence has an increased hardware complexity. We consider a partially halfduplex implementation of the proposed scheme in [13].

Fig. 4a compares ANC-FD with ANC-STBC and ANCOFDM in a time-varying channel for various fade rates $\left(f_{d} T_{s}\right)$ where $f_{d}$ is the Doppler frequency shift in Hz. With the assumed parameters and setting $D_{2 B}=286$, the minimum CP length for ANC-FD is $N_{C P}=184$. Moreover, the effective delay for ANC-FD is $D_{A B}=286$ samples (equivalent to 95.334

\footnotetext{
${ }^{1}$ The detailed derivation of the PEP upper bound is not included in the paper due to space limitations.

${ }^{2}$ These channels are defined by $\left\{\sigma_{h_{i r, l}}\right\}_{l \in\left\{1,2, \ldots, L_{h_{i}}\right\}}=[0.4714,0.2828$, $0.3771,0.1886,0.1414], i \in\{A, B\}, r \in\{1,2\}$ corresponding to the taps with delays $[1.67 \mathrm{~ms}, 2.67 \mathrm{~ms}, 3.67 \mathrm{~ms}, 7 \mathrm{~ms}, 20 \mathrm{~ms}]$, respectively.
} 
ms) which means that $B D_{A B}=1$ and $d_{A B}=38$. On the other hand, for the ANC-STBC and ANC-OFDM systems, the minimum CP length at each phase is 346 samples which means that the effective transmission of $N=64$ data samples using either ANC-STBC or ANC-OFDM requires 820 samples compared to only 248 samples using our ANC-FD systems.

Fig. 4a clearly shows that our proposed scheme performs better than ANC-STBC and ANC-OFDM for the three values of the fade rate due to having a delay-independent CP. For instance, when the channel is quasi-static $\left(f_{d} T_{s}=0\right)$, ANCFD outperforms ANC-STBC and ANC-OFDM by about 11 $\mathrm{dB}$ and $14 \mathrm{~dB}$, respectively, at a BER of about $10^{-2}$. Also, as expected, the performance of ANC-FD when the channel is quasi-static is at its best and degrades when the fading becomes faster as more ICI is experienced and the approximation that was adopted in (4) by ignoring the off-diagonal elements of the matrices $H_{d f, A r B}^{(m)}$ becomes inaccurate.

Fig. $4 \mathrm{~b}$ shows the BER versus the delay $\left(D_{A B}\right)$ for the quasi-static case $\left(f_{d} T_{s}=0\right)$. Here, we set a fixed SNR of 24 $\mathrm{dB}$ and vary $D_{2 B}$ from 0 to 480 . Clearly, the performances of ANC-STBC and ANC-OFDM suffer greatly due to the increase in delay. However, ANC-FD shows robustness against asynchrony since its performance is unaffected by the increase in delay. For instance, at a delay of $160 \mathrm{~ms}$ (may be observed in UWA communications [14]), a performance of about two orders of magnitude in the error rate is observed. It is also noted that the ANC-STBC performs better than the ANC-FD for small delay values (less than $10.2 \mathrm{~ms}$ for this example).

\section{Conclusions}

In this paper, we have considered a dual-relay TWR system in a doubly selective fading environment in which large delays are experienced with possible applications in UWA channels. We have proposed a spectrally-efficient scheme in which the $\mathrm{CP}$ length is independent from the delays experienced. An important aspect of our scheme is that it not only solves the large delay problem but it also harnesses the inherent delay diversity in the signal structure. Through simulations, we have illustrated the advantages of the proposed scheme compared to other existing solutions.

\section{REFERENCES}

[1] M. Stojanovic and J. Preisig, "Underwater acoustic communication channels: Propagation models and statistical characterization," IEEE Communications Magazine, vol. 47, no. 1, pp. 84-89, January 2009.

[2] S. Zhang, S. chang Liew, and P. P. Lam, "Physical-layer network coding," in ACM Mobicom, 2006.

[3] L. Lu, T. Wang, S. C. Liew, and S. Zhang, "Implementation of physical-layer network coding," in 2012 IEEE International Conference on Communications, June 2012, pp. 4734-4740.

[4] F. Rossetto and M. Zorzi, "A practical architecture for OFDMbased decode-and-forward physical layer network coding," IEEE Transactions on Signal Processing, vol. 60, no. 9, pp. 4747-4757, 2012.

[5] G. Bartoli, R. Fantacci, D. Marabissi, and R. Simoni, "Physical layer network coding in multipath channel: effective precodingbased transmission scheme," in 2011 IEEE Global Telecommunications Conference, Dec. 2011, pp. $1-5$.

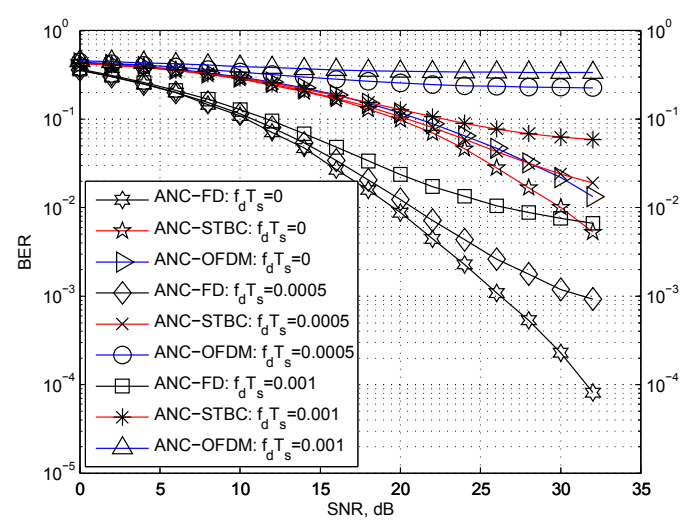

(a) Fixed delay of $D_{A B}=286$ samples.

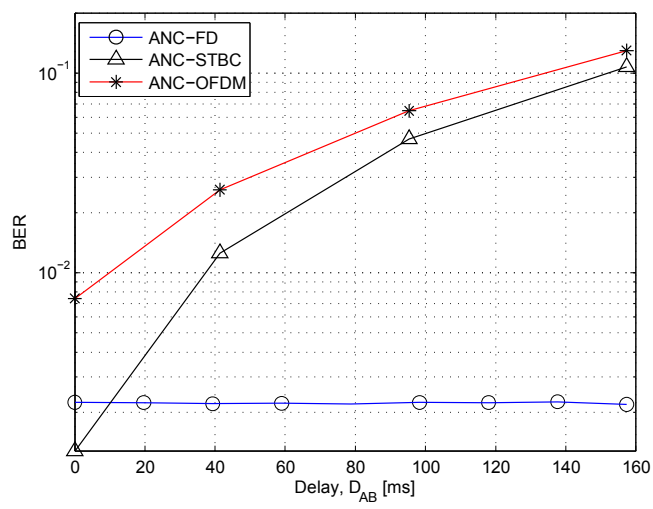

(b) Fixed SNR of $24 \mathrm{~dB}$.

Fig. 4: BER performance comparison between the proposed ANCFD scheme and two existing schemes $[3,6]$.

[6] Z. Li, X.-G. Xia, and B. Li, "Achieving full diversity and fast ML decoding via simple analog network coding for asynchronous two-way relay networks," IEEE Transactions on Communications, vol. 57, no. 12, pp. 3672 -3681, Dec. 2009.

[7] Y. Yao and X. Dong, "Optimal timing at the relay in OFDM based two way relay systems," Wireless Personal Communications, pp. 1-15, 2013.

[8] Z. Wang, J. Huang, S. Zhou, and Z. Wang, "Iterative receiver processing for OFDM modulated physical-layer network coding in underwater acoustic channels," IEEE Transactions on Communications, Apr. 2013.

[9] M. Rahmati and T. M. Duman, "Achieving delay diversity in asynchronous underwater acoustic (UWA) cooperative communication systems," IEEE Transactions on Wireless Communications, vol. 13, no. 3, pp. 1367-1379, March 2014.

[10] S. Katti, S. Gollakota, and D. Katabi, "Embracing wireless interference: analog network coding," Wireless Networks, vol. 37, no. 4, pp. 397-408, 2007.

[11] P. Schniter, "Low-complexity equalization of OFDM in doubly selective channels," IEEE Transactions on Signal Processing, vol. 52, no. 4, pp. 1002-1011, 2004.

[12] B. Lu, X. Wang, and K. Narayanan, "LDPC-based spacetime coded OFDM systems over correlated fading channels: performance analysis and receiver design," IEEE Transactions on Communications, vol. 50, no. 1, pp. 74-88, Jan 2002.

[13] A. Salim and T. M. Duman, "A delay-tolerant asynchronous two-way-relay system over doubly-selective fading channels," submitted for publication, 2014.

[14] M. K. Park and V. Rodoplu, "UWAN-MAC: An energy-efficient MAC protocol for underwater acoustic wireless sensor networks," IEEE Journal of Oceanic Engineering, vol. 32, no. 3, pp. 710-720, July 2007. 